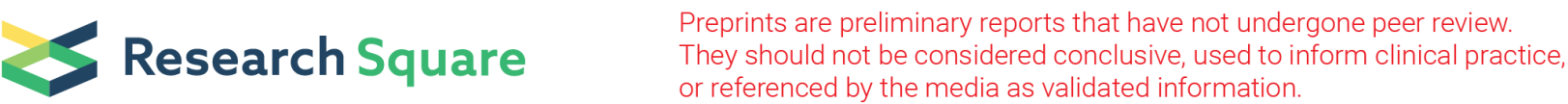

\section{Reactivity and reproducibility of accelerometer- based sedentary behavior and physical activity in two measurement periods: Results of a feasibility randomized-controlled study}

Antje Ullrich ( $\square$ antje.ullrich@med.uni-greifswald.de )

Institute of Social Medicine and Prevention, University Medicine Greifswald https://orcid.org/00000002-3905-858X

\section{Sophie Baumann}

Technische Universitat Dresden

Lisa Voigt

Universitatsmedizin Greifswald

Ulrich John

Universitatsmedizin Greifswald

Sabina Ulbricht

Universitatsmedizin Greifswald

Research article

Keywords: sitting position, physical health, exercise, accelerometry, measurement reactivity, adults

Posted Date: October 2nd, 2019

DOl: https://doi.org/10.21203/rs.2.15452/v1

License: (c) (i) This work is licensed under a Creative Commons Attribution 4.0 International License.

Read Full License 


\section{Abstract}

Background: The aims of the study were to investigate measurement reactivity in sedentary behavior (SB), physical activity (PA), and accelerometer wear time in two measurement periods, to examine the reproducibility of these outcomes and to quantify measurement reactivity as a confounder for the reproducibility of SB and PA data.

Methods: A total of 136 participants ( $65 \%$ women, mean age $=54.6$ years, study period $02 / 2015$ to 08/2016) received 7-day accelerometry at baseline and after 12 months. Latent growth models were used to identify measurement reactivity in each period. Intraclass correlations (ICC) were calculated to examine the reproducibility using two-level mixed-effects linear regression analyses.

Results: At both measurements periods, participants increased time spent in SB ( $b=2.3 \mathrm{~min} / \mathrm{d} ; \mathrm{b}=3.8$ $\mathrm{min} / \mathrm{d})$, reduced time spent in light $P A(b=2.0 \mathrm{~min} /$ day; $b=3.3 \mathrm{~min} / \mathrm{d})$, but did not change moderate-tovigorous PA. Participants reduced accelerometer wear time $(b=4.6 \mathrm{~min} / \mathrm{d})$ only at baseline. The ICC coefficients ranged from $0.42(95 \% \mathrm{Cl}=0.29-0.57)$ for accelerometer wear time to $0.70(95 \% \mathrm{Cl}=0.61-0.78)$ for moderate-to-vigorous PA. In none of the regression models, a reactivity indicator was identified as a confounder for the reproducibility of SB and PA data.

Conclusions: The results show that measurement reactivity differentially influences SB and PA in two measurement periods. Although 7-day accelerometry seems to be a reproducible measure of SB and PA, our findings highlight the importance of accelerometer wear time as a crucial confounder when using accelerometry in monitoring SB and PA, planning interventions, and analyzing SB and PA data.

Clinical trial registration number: NCT02990039 (retrospectively registered); December 12, 2016

\section{Background}

In order to determine the level of sedentary behavior (SB) and physical activity (PA), to understand their relationship with health, and to evaluate the efficacy of behavioral interventions, an accurate measure of $\mathrm{SB}$ and PA is required. ${ }^{1}$ This is challenging because both behaviors are characterized by considerable inter- and intraindividual variability. ${ }^{2}$ Day-to-day variability around a mean level is a natural part of SB

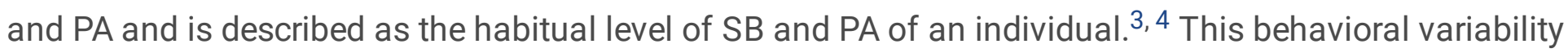
needs to be carefully studied because it has major implications for measurement and conclusions, drawn from data analysis. ${ }^{4}$

Accelerometry is frequently used to assess SB and PA. The reproducibility of accelerometer-based measures is subject to human-related sources of bias. ${ }^{5}$ Participants can influence accurate data measurement by changing their behavior when wearing an accelerometer, even if they do not intend to do so. ${ }^{6}$ The motivation to be physically more active than usual due to wearing such a device or social desirability may be reasons for the presence of accelerometer measurement reactivity (AMR). ${ }^{7}$ So far, the 
evidence on AMR among adults is inconsistent. ${ }^{8-14} \mathrm{Few}$ studies investigated the influence of AMR on the entire intensity spectrum from SB to moderate-to-vigorous PA (MVPA). ${ }^{14}$ To our best knowledge, none of these studies has examined whether and to what extent AMR occurs in more than one measurement period.

The vast majority of studies applied a 7-day accelerometry protocol to determine habitual levels of SB and PA. ${ }^{15}$ However, this approach raises the question whether this length of monitoring protocol is sufficient to reflect the mean level on the entire intensity spectrum from SB to MVPA. Reliable data depend on the variability within a person's daily activity pattern, ${ }^{3}$ especially for more than one measurement period. ${ }^{16}$ Moreover, the potential impact of AMR on the reproducibility of SB and PA data was not investigated so far.

Therefore, the present study has two aims. First, to examine AMR over two measurement periods indicated by systematic changes in SB, light PA (LPA), MVPA, and accelerometer wear time. Second, to investigate the reproducibility of these outcomes and to quantify whether AMR should be considered as a relevant confounder to estimate the reproducibility of SB and PA data.

\section{Methods}

Study participants: From a sample of 1165 individuals aged between 40 and 70 years who had been recruited in general medical practices, job agencies, or via a health insurance company between June 2012 and December 2013, 95\% gave written consent to be contacted again. Of those, a total of 401 persons were offered participation in a randomized-controlled study that aimed to assess the feasibility of a brief tailored letter intervention to increase PA and to reduce SB in leisure time. The design and participant flow were described in detail elsewhere. ${ }^{17}$

SB, LPA, and MVPA were measured with accelerometry at baseline $(n=175)$ and after 12 months $(n=$ $165,94 \%)$. Two participants at each measurement period were excluded due to missing accelerometer data (excluded, $n=4$ ). In addition, we analyzed data only among those who have worn the accelerometer $\geq 10$ hours per day (h/day) on $\geq 5$ days including at least one weekend day (excluded, $n=25$ ). The final sample size comprised 136 participants.

Procedure: At baseline and after 12 months, all participants underwent the following procedure: (1) cardiovascular health program including blood sample taking and standardized measurement of blood pressure, waist circumference, body weight, and height at the cardiovascular examination center of the University Medicine Greifswald; (2) self-administered assessments of socio-demographics, SB, and PA; (3) wearing an accelerometer for seven consecutive days; and (4) protocolling daily working hours over the monitoring period.

Study participants were instructed to wear the accelerometer on their right hip with an elastic band, to start the day after the cardiovascular health program in the morning after getting dressed, and to take it 
off during night's sleep and water activities. All participants were informed that PA would be recorded for seven days.

After baseline assessments, participants were randomized into an assessment-only group $(n=85)$ or an intervention group $(n=90)$. Additionally, for all participants, self-administered assessments of SB and PA were conducted at month $1,3,4$, and 6 after baseline. Only individuals of the intervention group received up to three letters tailored to their self-reported SB and PA at month 1, 3, and 4 . The study was conducted between February 2015 and August 2016 and was approved by the clinical ethical committee of the University Medicine Greifswald (protocol number BB 002/15a).

Measures: Accelerometer-based data were assessed using a tri-axial ActiGraph Model GT3X+ accelerometer (Pensacola, FL). The accelerometers were initialized at a sampling rate of $100 \mathrm{Hertz}$ and raw data were integrated into 10-second epochs. Data from the vertical axis were used. For statistical analysis, data from the accelerometers were downloaded and processed using ActiLife software (Version 6.13.3; ActiGraph).

Time spent in SB, LPA, MVPA, and wearing the accelerometer was determined by minutes per day (min/day). Non-wear time was calculated by the Troiano algorithm, defined as at least 60 consecutive minutes of zero activity intensity counts, with allowance for $\leq 2$ minutes of counts (counts/min) between 0 and 100. To identify the time spent in different intensities of PA, we used cut points according to different intensity threshold criteria. ${ }^{18}$ Values $<100$ counts/min were determined as SB, values between 100 and 2019 counts/min as LPA, and values $\geq 2020$ counts/min as MVPA.

Sex, age, and years of school education ( $<10$ years/ 10 to 11 years/ $\geq 12$ years) were obtained by a selfadministrative questionnaire. In addition, study group (assessment-only group/ intervention group), time (baseline/ after 12 months), recruitment site (general practice/ job center/ health insurance), first day of measurement (weekday/ weekend day), ${ }^{19}$ season of data collection (winter/ spring/ summer), ${ }^{20}$ and the average number of working hours ${ }^{4}$ on each day the accelerometer was worn were included as covariates.

Statistical analyses: We decided to include data from both study groups as all participants received almost the same assessment procedure, the feasibility study was not powered to detect differences between assessment-only group and intervention group, and previously published data revealed that there were no differences in self-reported PA and SB between groups after 12 months. ${ }^{17}$

SB, LPA, and accelerometer wear time were approximately normally distributed, thus untransformed values were used for analyses. To account for their right-skewed distributions, MVPA data were square root transformed. For all analyses, $p$-values below 0.05 were considered statistically significant.

Latent growth models were used to investigate AMR for both measurement periods. ${ }^{21}$ In line with Baumann et al., ${ }^{14}$ time spent in SB, LPA, MVPA, and wearing the accelerometer on each of the seven days of measurement was represented by seven observed indicators of these continuous latent variables (growth factors). The indicators were regressed on latent growth factors representing trajectories of 
outcomes over a week. ${ }^{21} \mathrm{~A}$ maximum likelihood estimator with robust standard errors was used. The shape of the growth curves was determined by time scores defined in the measurement model of the growth factors and matched with the observed day number of the measurement week. To specify nonlinear growth curves, an overall change function (e.g., linear, quadratic, cubic) was fitted to the sample by adding quadratic and cubic slopes of time scores to the models. Rescaled Likelihood Ratio Tests were used to test whether higher order functions of time scores and free growth factor variances were required. ${ }^{22}$ Accelerometer wear time and working hours may have been taken into account as timevarying covariates that were specified to predict outcomes at the corresponding day of measurement. Non-zero time trends in the outcomes over the days of measurement would imply reactivity. In the models, the slope factor was freely estimated if appropriate and treated as a reactivity indicator reflecting the individual average change in outcome over time. Therefore, the factor scores of outcomes were saved and included as a reactivity indicator in further analysis. Statistical analyses were performed using Mplus version $7.316 .^{22}$

For each outcome, the average of the 7 days of measurement was calculated. Two-level (individual and time) mixed-effects linear regression analyses were performed to assess changes in accelerometer-based outcomes from baseline to 12 months apart, including a random intercept for subjects. All regression models were adjusted for sex, age, education, study group, time, recruitment site, first day of measurement, and season of data collection. In addition, we added the individual average value of accelerometer wear time, the reactivity indicator of accelerometer wear time, the reactivity indicator of the respective SB and PA outcome, and a combination of these factors as potential covariates step-by-step.

We used intraclass correlation (ICC) coefficients to decide which model for each outcome was most appropriate. The ICC is a measure of reproducibility of replicate measures from the same subject. ${ }^{23}$ The ICC coefficient is classified as follows: less than 0.4 indicates poor, between 0.4 and 0.75 fair to good, and 0.75 or more excellent reproducibility. ${ }^{23}$ To illustrate the agreement between both measurement periods and to estimate the limits of agreement interval (95\% Confidence Interval, $\mathrm{Cl}$ ), Bland Altman plots were applied. Statistical analyses were performed using Stata/ SE version 14.2. ${ }^{24}$

\section{Results}

Sample characteristics: In our sample, the mean age was 54.6 years and $65.4 \%$ were women. The majority of the participants attended school 10 to 11 years (70.4\%). Table 1 provide data on time spent in SB, LPA, and MVPA and wearing the accelerometer from baseline to 12 months.

Accelerometer measurement reactivity: As shown in Figure 1, at baseline and after 12 months, participants increased time spent in SB by $2.3 \mathrm{~min} /$ day and by $3.8 \mathrm{~min} /$ day and reduced time spent in LPA by $2.0 \mathrm{~min} /$ day and by $3.3 \mathrm{~min} /$ day, respectively. Neither at baseline nor after 12 months participants significantly changed time they spent in MVPA. Participants significantly reduced the accelerometer wear time by $4.6 \mathrm{~min} /$ day at baseline. After 12 months, participants did not significantly change accelerometer wear time. The reactivity indicators of accelerometer wear time $(p<0.001), \operatorname{SB}(p=0.008)$, and LPA $(p=$ 
0.006) significantly differed from baseline to 12 months apart. Results of baseline and after 12 months parameter estimates for latent growth models of SB, LPA, MVPA, and accelerometer wear time are shown in additional file 1 (Tables S1/ S2).

- Figure 1 -

Reproducibility of accelerometer-based outcomes: The ICC $(95 \% \mathrm{Cl})$ ranged from fair to good reproducibility of the outcomes; coefficient values lied between $0.41(0.28,0.56)$ for accelerometer wear time and $0.70(0.61,0.78)$ for LPA (additional file 2; Table S3). The regression model additionally adjusted for the average value of accelerometer wear time was most appropriate for SB $(I C C=0.68)$, for LPA (ICC = $0.68)$, and for MVPA $(I C C=0.70)$. For accelerometer wear time, none of the additional adjustments were appropriate $(I C C=0.42)$.

The Bland Altman plots visualize the agreement between the two measurement periods, as a function of the mean of these two measurement periods (additional file 3; Figure S1). For accelerometer wear time values of ten participants (7.4\%), for SB values of nine participants $(6.6 \%)$, for LPA values of eleven participants $(8.1 \%)$, and for MVPA values of four participants $(2.9 \%)$ lied outside the limits of agreement.

Using the most appropriate regression model for each outcome, neither the time spent in SB, LPA, MVPA, nor accelerometer wear time significantly declined or increased over time (Table 2). In all SB and PA regression models, the average value of accelerometer wear time was a significant confounder.

\section{Discussion}

The present study has two main findings. First, there was a significant linear trend in SB and LPA time series as an indicator of AMR for baseline and 12 months apart, whereas MVPA does not seem to be affected by AMR. Further, the systematic changes within accelerometer wear time differed between the two measurement periods. Second, our results showed that the time spent in SB, LPA, and MVPA and wearing the accelerometer are fairly stable between the two measurement periods. AMR operationalized by a reactivity indicator could not be identified as a relevant confounder for the estimation of the reproducibility of SB and PA data.

In line with previous literature, the results of the present study indicate that persons change SB and PA in the presence of an accelerometer. ${ }^{9,10,12-14}$ This study adds to the literature by showing that AMR differentially influences SB and PA in two measurement periods depending on the intensity level of PA. In line with Baumann and colleagues, participants appeared to replace SB with LPA. ${ }^{14}$ In addition, this study showed that the trend of both time series seems to be the same over the two measurement periods. Evidence in recent studies showed that LPA includes standing and walking at a light pace, and therefore logically tends to be more related to SB activities than MVPA. ${ }^{25}$ Moreover, adults may not move enough, knowing well that they should move more. ${ }^{6}$ Therefore, attempts are made to reduce this potential discrepancy when wearing a device, ${ }^{7}$ and mostly replace SB with LPA. For behavioral interventions, it can 
be assumed that AMR has a similar impact on SB and LPA over time. The best advice for reducing bias due to AMR is to consider this in the planning of the study. ${ }^{26}$ In case of brief interventions, measurement periods in terms of duration and frequency as well as number of methods used to assess SB and PA should be balanced to identify potential intervention effects.

Furthermore, our results showed that MVPA seemed to be less altered by AMR than SB and LPA. This could be because of MVPA typically requires more planning and is likely to be more structured than lighter physical activities. Furthermore, the ICC coefficient value showed that MVPA was the most reproducible of all outcomes using a 7-day accelerometry monitoring. This seems to be in contrast to recent findings. It has been argued that MVPA is less predictable on a day-to-day basis and requires more monitoring days to determine reproducible habitual behavior. ${ }^{3,4}$ Further, a recently published study that examined the reproducibility in accelerometer-assessed SB and PA reported the highest ICC values for SB. ${ }^{16}$ It should be mentioned that in both studies the samples differed from our sample. While KozeyKeadle and colleagues investigate reproducibility in older adult women with a mean age of 71 years, Bergman used a convenience sample consisting of younger university students and staff. ${ }^{3,16}$ It should be noted that ICC coefficients are constrained by the sample in which it was collected, because the magnitudes of intra- and inter-individual variability in SB and PA depends on the characteristics of the study sample. ${ }^{27}$ Although the results reported in this study indicate that a 7-day accelerometry monitoring seems to be a reproducible measure of SB and PA, Bland Altman plots showed that there is a high intraindividual variability in SB and PA data. Therefore, the findings should be interpreted with caution and future studies are required to verify the findings of the present study using a larger sample of adults.

Our findings on accelerometer wear time highlight the importance of this factor. The value for the ICC coefficient of accelerometer wear time was the lowest of all outcomes. In terms of AMR, the systematic changes in time of wearing the accelerometer differed in the magnitude between the repeated measurements. In this regard, providing clear instructions to constantly wear the device to provide valid data at each measurement time point seems to be of great importance for the compliance of participants over time. ${ }^{28}$ Accelerometer wear time was the only relevant predictor in all SB and PA regression models, whereas AMR operationalized by a reactivity indicator could not be identified as a relevant confounder for the reproducibility of PA and SB data. In line with other studies, this indicates that accelerometer wear time should be considered as a crucial confounder in data analysis of accelerometer data. ${ }^{27,29}$

To reduce AMR and, on the other hand, increase the precision of the measurement, a longer measurement period may be reasonable. Although a longer measurement period might improve reproducibility, the burden for study participants and study feasibility should be considered because it influences the response rate and compliance. ${ }^{27}$

Some limitations have to be discussed. First, the sample size was small and therefore, our findings may suffer from a lack of power when estimating AMR. Second, generalizability of our results may be compromised due to selection bias. Our sample was highly motivated and compliant, with an average wear time of $13 \mathrm{~h} /$ day. As reactivity may be most pronounced in people with a high motivation to change 
behavior, AMR may have been overestimated in our study. Third, we used hip-worn accelerometers that cannot differentiate between sitting and standing still because movement is determined by acceleration rather than body posture. ${ }^{30}$ Thus, our findings regarding SB and LPA can give a distorted picture on AMR. Finally, using the slope factor as a reactivity indicator to operationalize AMR is just one of several other ways to account for AMR for estimating the reproducibility of SB and PA data.

\section{Conclusions}

In conclusion, the results of the present study show that AMR differentially influences SB and PA in two measurement periods. Although a 7-day accelerometry monitoring seems to be a reproducible measure of $\mathrm{SB}$ and $\mathrm{PA}$, our findings highlight the importance of accelerometer wear time as a crucial confounder when using accelerometer in monitoring SB and PA, planning interventions, and analyzing SB and PA data.

\section{List Of Abbreviations}

AMR: Accelerometer measurement reactivity; Cl: Confidence Interval; ICC: intraclass correlation; LPA: light physical activity; MVPA: moderate-to-vigorous physical activity; PA: physical activity; SB: sedentary behavior

\section{Declarations}

\section{Acknowledgements}

The authors wish to thank funders, supporters, and participants of the study.

\section{Funding}

This study was funded by the Federal Ministry of Education and Research as part of the German Centre of Cardiovascular Research, DZHK (grant no. 81/Z540100152). The DZHK had no direct role in the development of methodology, the acquisition, analysis, and interpretation of data or in writing the manuscript.

\section{Availability of data and materials}

The datasets generated and/or analyzed during the current study are not publicly available due to restrictions associated with anonymity of participants but are available from the corresponding author on reasonable request. Researchers requesting the data will be required to sign a contract ensuring data usage in compliance with the statement given in the informed consent procedure and with the German data protection law, that the data will not be transferred to others, and that the data will be deleted after 
the intended analysis has been completed. To comply with the statement given in the informed consent, the use of the data is restricted to research related to cardiovascular-research questions. We cannot ensure to prevent use for other purposes when uploading the data for public access.

\section{Authors' contributions}

UJ and SU contributed to the conception or design of the study. AU, SB, LV, UJ, and SU contributed to the acquisition, analysis, or interpretation of data for the work. AU drafted the manuscript. All authors critically revised the manuscript and gave final approval.

\section{Ethics approval and consent to participate}

All procedures performed in studies involving human participants were in accordance with the ethical standards of the institutional and/or national research committee and with the 1964 Helsinki Declaration and its later amendments or comparable ethical standards.

This study was approved by the clinical ethical committee of the University Medicine Greifswald, Germany (protocol number BB 002/15a) and retrospectively registered to the ClinicalTrials.gov Protocol Registration and Results System (Clinical trial registration number: NCT02990039). Informed written consent was obtained from all individual participants included in the study.

\section{Consent for publication}

Not applicable.

\section{Competing interests}

The authors declare that they have no competing interests.

\section{References}

1.De Vries SI, Van Hirtum HW, Bakker I, et al. Validity and reproducibility of motion sensors in youth: a systematic update. Med Sci Sports Exerc 2009;41:818-827. 2009/03/12. DOI: 10.1249/MSS.0b013e31818e5819.

2.Baranowski T, Masse LC, Ragan B, et al. How many days was that? We're still not sure, but we're asking the question better! Med Sci Sports Exerc 2008;40:S544-549. 2008/07/17. DOI:

10.1249/MSS.0b013e31817c6651. 
3.Bergman P. The number of repeated observations needed to estimate the habitual physical activity of an individual to a given level of precision. PLoS One 2018;13:e0192117. 2018/02/02. DOI:

$10.1371 /$ journal.pone.0192117.

4.Pedersen ES, Danquah IH, Petersen $\mathrm{CB}$, et al. Intra-individual variability in day-to-day and month-tomonth measurements of physical activity and sedentary behaviour at work and in leisure-time among Danish adults. BMC Public Health 2016;16:1222. 2016/12/05. DOI: 10.1186/s12889-016-3890-3.

5.Pedisic $Z$ and Bauman A. Accelerometer-based measures in physical activity surveillance: current practices and issues. Brit J Sport Med 2015;49:219-223. 2014/11/06. DOI: 10.1136/bjsports-2013093407.

6.Vanhelst J, Beghin L, Drumez E, et al. Awareness of wearing an accelerometer does not affect physical activity in youth. BMC Med Res Methodol 2017;17:99. 2017/07/12. DOI: 10.1186/s12874-017-0378-5.

7.Dossegger A, Ruch N, Jimmy G, et al. Reactivity to accelerometer measurement of children and adolescents. Med Sci Sports Exerc 2014;46:1140-1146. 2013/11/14. DOI:

$10.1249 / \mathrm{mss} .0000000000000215$.

8.Davis RE and Loprinzi PD. Examination of Accelerometer Reactivity Among a Population Sample of Children, Adolescents, and Adults. J Phys Act Health 2016;13:1325-1332. 2016/10/25. DOI:

10.1123/jpah.2015-0703.

9.van Sluijs EM, van Poppel MN, Twisk JW, et al. Physical activity measurements affected participants' behavior in a randomized controlled trial. J Clin Epidemio/ 2006;59:404-411. 2006/03/22. DOI:

10.1016/j.jclinepi.2005.08.016.

10.Motl RW, McAuley E and Dlugonski D. Reactivity in baseline accelerometer data from a physical activity behavioral intervention. Health Psychol 2012;31:172-175. 2011/10/26. DOI: 10.1037/a0025965.

11.Behrens TK and Dinger MK. Motion sensor reactivity in physically active young adults. Res $Q$ Exerc Sport 2007;78:1-8. 2007/05/08. DOI: 10.1080/02701367.2007.10762229.

12.Eisenberg MH, Phillips LA, Fowler L, et al. The Impact of E-diaries and Accelerometers on Young Adults' Perceived and Objectively Assessed Physical Activity. Psychol Sport Exerc 2017;30:55-63. 2017/10/03. DOI: 10.1016/j.psychsport.2017.01.008.

13.Vandoni M, Correale L, Del Bianco M, et al. Does reactivity to accelerometers occur in a single trial? Brief report in a sample of young adults. J Health Psychol 2017;22:1458-1462. 2016/02/18. DOl: $10.1177 / 1359105316628758$.

14.Baumann S, Gross S, Voigt L, et al. Pitfalls in accelerometer-based measurement of physical activity: The presence of reactivity in an adult population. Scand J Med Sci Sports 2018;28:1056-1063 2017/09/19. DOI: 10.1111/sms.12977. 
15.Migueles JH, Cadenas-Sanchez C, Ekelund U, et al. Accelerometer Data Collection and Processing Criteria to Assess Physical Activity and Other Outcomes: A Systematic Review and Practical Considerations. Sports Med 2017;47:1821-1845. 2017/03/18. DOI: 10.1007/s40279-017-0716-0.

16.Kozey-Keadle S, Shiroma EJ, Kamada M, et al. Reproducibility of Accelerometer-Assessed Physical Activity and Sedentary Time. Am J Prev Med 2017;52:541-548. 2017/01/08. DOI:

10.1016/j.amepre.2016.11.010.

17.Voigt L, Baumann S, Ullrich A, et al. The effect of mere measurement from a cardiovascular examination program on physical activity and sedentary time in an adult population. BMC Sports Sci Med Rehabil 2018;10:1. 2018/02/08. DOI: 10.1186/s13102-018-0090-8.

18.Troiano RP, Berrigan D, Dodd KW, et al. Physical activity in the United States measured by accelerometer. Med Sci Sports Exerc 2008;40:181-188. 2007/12/20. DOI:

10.1249/mss.0b013e31815a51b3.

19.McVeigh JA, Winkler EA, Howie EK, et al. Objectively measured patterns of sedentary time and physical activity in young adults of the Raine study cohort. Int J Behav Nutr Phys Act 2016;13:41. 2016/03/25. DOI: $10.1186 / \mathrm{s} 12966-016-0363-0$.

20.Tucker $P$ and Gilliland J. The effect of season and weather on physical activity: a systematic review. Public Health 2007;121:909-922. 2007/10/09. DOI: 10.1016/j.puhe.2007.04.009.

21.Wang J and Wang X. Structural equation modeling: Applications using Mplus. West Sussex, UK: John Wiley \& Sons, 2012.

22.Muthen LK and Muthen BO. Mplus User's Guide. Los Angeles, CA: Muthén \& Muthén, 1998-2012.

23.Rosner B. Fundamentals of Biostatistics. 8th Edition ed.: Duxbury Press; Belmont, CA, 2016.

24.StataCorp. Stata Statistical Software: Release 14. College Station, TX: StataCorp LP, 2015.

25.Mansoubi M, Pearson N, Biddle SJH, et al. The relationship between sedentary behaviour and physical activity in adults: A systematic review. Prev Med 2014;69:28-35. DOI:

http://dx.doi.org/10.1016/j.ypmed.2014.08.028.

26.French DP and Sutton S. Reactivity of measurement in health psychology: how much of a problem is it? What can be done about it? Br J Health Psychol 2010;15:453-468. 2010/03/09. DOl: $10.1348 / 135910710 \times 492341$.

27.Aadland E and Ylvisaker E. Reliability of Objectively Measured Sedentary Time and Physical Activity in Adults. PLoS One 2015;10:e0133296. 2015/07/21. DOI: 10.1371/journal.pone.0133296. 
28.Matthews $\mathrm{CE}$, Hagstromer M, Pober DM, et al. Best practices for using physical activity monitors in population-based research. Med Sci Sports Exerc 2012;44:S68-76. 2011/12/23. DOI:

10.1249/MSS.0b013e3182399e5b.

29. Herrmann SD, Barreira TV, Kang M, et al. Impact of accelerometer wear time on physical activity data: a NHANES semisimulation data approach. Brit J Sport Med 2014;48:278-282. 2012/09/01. DOI: 10.1136/bjsports-2012-091410.

30. Heesch KC, Hill RL, Aguilar-Farias N, et al. Validity of objective methods for measuring sedentary behaviour in older adults: a systematic review. Int J Behav Nutr Phys Act 2018;15:119. 2018/11/28. DOI: 10.1186/s12966-018-0749-2.

\section{Tables}

Table 1 Descriptive characteristics of the study sample $(n=136)$. 


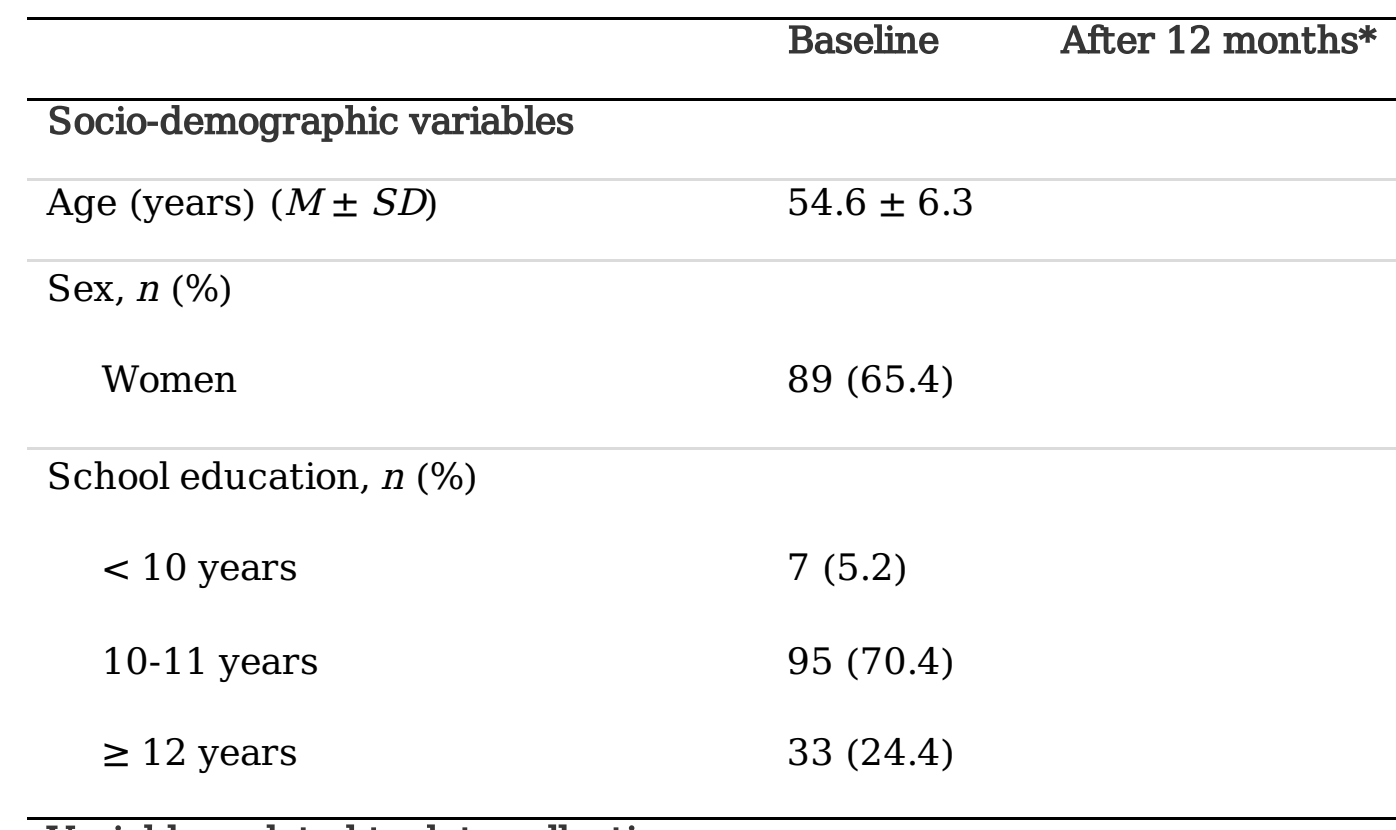

\section{Variables related to data collection}

Recruitment site, $n(\%)$

General practice

$54(39.7)$

Job center

$24(17.6)$

Health insurance company

$58(42.7)$

First day of measurement, $n(\%)$

Weekday

$113(83.1) \quad 107(78.7)$

Season, $n(\%)$

Winter

$9(6.6)$

$7(5.2)$

Spring

$118(86.8) \quad 120(88.2)$

Summer

9 (6.6)

9 (6.6)

Working hours, h/day $(M \pm S D) \quad 4.1 \pm 3.0 \quad 3.5 \pm 3.0$

Accelerometer-based variables, $\min /$ day $(M \pm S D)$

Accelerometer wear time

$880.8 \pm 85.9 \quad 864.8 \pm 93.6$

Sedentary behaviour

$602.6 \pm 96.6 \quad 593.8 \pm 99.2$

Light physical activity

$230.9 \pm 60.1227 .5 \pm 57.9$

Moderate-to-vigorous physical activity

$47.3 \pm 24.4$

$43.5 \pm 23.0$

$M$, mean; $S D$, standard deviation; min, Minutes; $\mathrm{h}$, hours. * One participant with missing values on accelerometer-based variables. 
Table 2 Results of multilevel mixed-effects linear regression models for minutes of sedentary behavior, light physical activity, moderate-to-vigorous physical activity, and accelerometer wear time $(n=136)$.

\begin{tabular}{|c|c|c|c|c|c|c|c|c|}
\hline & \multicolumn{2}{|c|}{ SB } & \multicolumn{2}{|c|}{ LPA } & \multicolumn{2}{|c|}{ MVPA } & \multicolumn{2}{|c|}{$\begin{array}{l}\text { Accelerometer } \\
\text { wear time }\end{array}$} \\
\hline & $\begin{array}{c}\beta[95 \% \\
C I]\end{array}$ & $p$ & $\begin{array}{c}\beta[95 \% \\
C I]\end{array}$ & $p$ & $\begin{array}{c}\beta[95 \% \\
C I]\end{array}$ & $p$ & $\begin{array}{c}\beta[95 \% \\
C I]\end{array}$ & $p$ \\
\hline \multirow{2}{*}{$\begin{array}{l}\text { Study group } \\
\text { (Ref. } \\
\text { Assessment-only } \\
\text { group) }\end{array}$} & 22.92 & 0.053 & -22.94 & 0.017 & 0.15 & 0.955 & 7.93 & 0.606 \\
\hline & $\begin{array}{l}{[-0.33,} \\
46.18]\end{array}$ & & $\begin{array}{c}{[-41.75} \\
-4.13]\end{array}$ & & $\begin{array}{c}{[-0.51} \\
0.54]\end{array}$ & & $\begin{array}{c}{[-22.21} \\
38.07]\end{array}$ & \\
\hline \multirow{2}{*}{$\begin{array}{l}\text { Time (Ref. } \\
\text { Baseline) }\end{array}$} & 9.50 & 0.184 & 2.84 & 0.620 & -0.29 & 0.061 & -20.13 & 0.096 \\
\hline & $\begin{array}{l}{[-4.52,} \\
23.52]\end{array}$ & & $\begin{array}{l}{[-8.41} \\
14.10]\end{array}$ & & $\begin{array}{c}{[-0.59} \\
0.01]\end{array}$ & & $\begin{array}{c}{[43.81} \\
3.54]\end{array}$ & \\
\hline \multirow{2}{*}{$\begin{array}{l}\text { Study group } \mathrm{x} \\
\text { time }\end{array}$} & -8.78 & 0.360 & 3.91 & 0.611 & 0.42 & 0.043 & 8.9 & 0.588 \\
\hline & $\begin{array}{c}{[27.55,} \\
10.00]\end{array}$ & & $\begin{array}{c}{[-11.16,} \\
18.98]\end{array}$ & & $\begin{array}{c}{[0.01} \\
0.82]\end{array}$ & & $\begin{array}{c}{[-23.41,} \\
41.33]\end{array}$ & \\
\hline \multirow{2}{*}{$\begin{array}{l}\text { Accelerometer } \\
\text { wear time }\end{array}$} & 0.72 & $<0.001$ & 0.20 & $<0.001$ & 0.00 & $<0.001$ & - & \\
\hline & $\begin{array}{l}{[0.64,} \\
0.80]\end{array}$ & & $\begin{array}{l}{[0.14,} \\
0.27]\end{array}$ & & $\begin{array}{l}{[0.00,} \\
0.01]\end{array}$ & & & \\
\hline
\end{tabular}

Notes: SB = Sedentary behavior; LPA = Light physical activity; MVPA = moderate-to-vigorous physical activity; $\beta$, Standardized regression coefficient; $C I$, Confidence interval; Interaction term (study group $\mathrm{x}$ time). All models were adjusted for sex, age, education, study group, time, first day of measurement, season of data collection, and recruitment site.

Figures 


\section{Baseline}

(A) Minutes of sedentary behavior

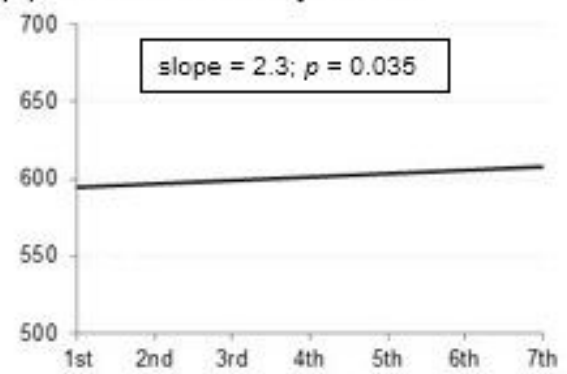

(B) Minutes of light physical activity

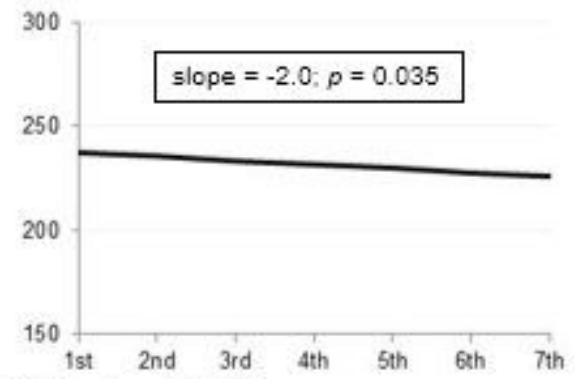

(C) Minutes of MVPA

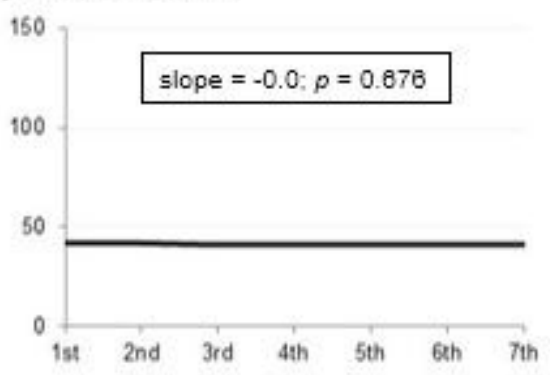

(D) Minutes of accelerometer wear time

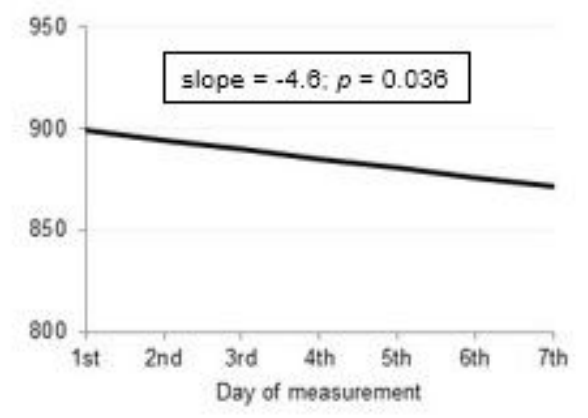

\section{After 12 months}
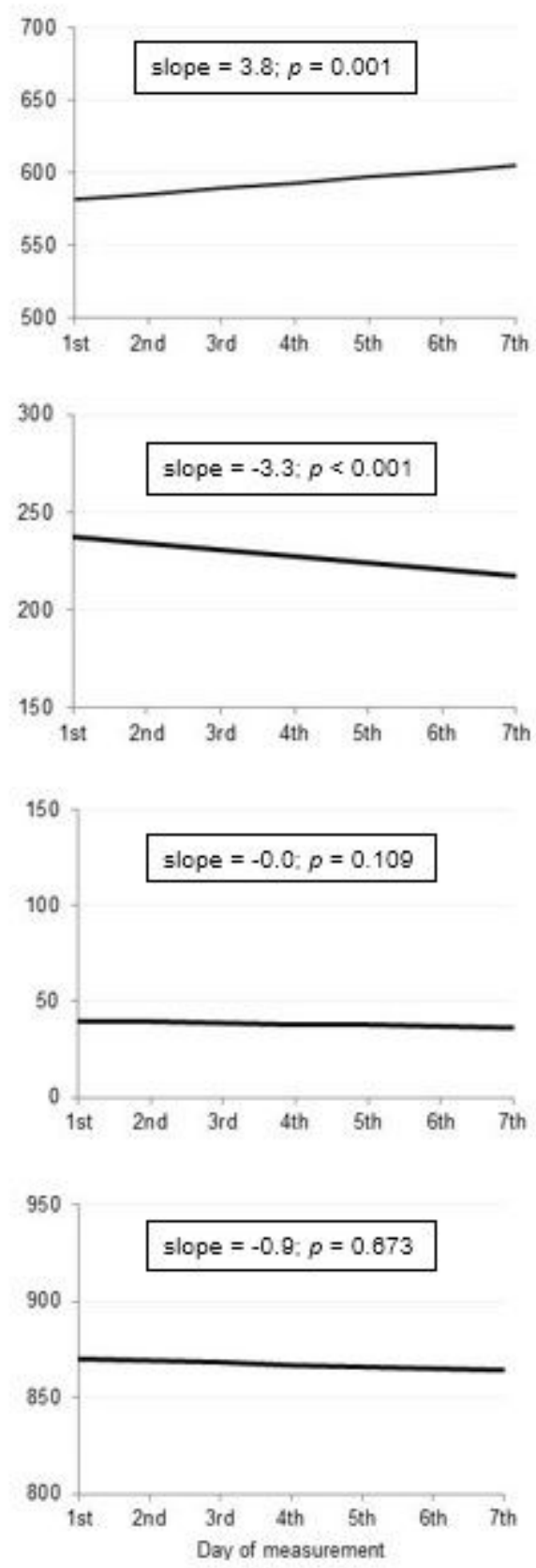

\section{Figure 1}

Estimated average linear growth curves for minutes spent in sedentary behavior (A), light physical activity (B), moderate-to-vigorous physical activity (MVPA) (C), and wearing the accelerometer (D) to baseline and after 12 months. Adjusted for accelerometer wear time and working hours if appropriate.

\section{Supplementary Files}

This is a list of supplementary files associated with this preprint. Click to download. 
- CONSORTextensionforPilotandFeasibility TrialsChecklist.doc

- MsUllrichAdditionalfiles.docx 